\title{
LRRK2: A Problem Lurking in Vesicle Trafficking?
}

\author{
Sybille Dihanich and Claudia Manzoni \\ Department of Molecular Neuroscience, UCL Institute of Neurology, WC1N 3BG, London, United Kingdom \\ Review of Piccoli et al.
}

Mutations in leucine-rich repeat kinase 2 (LRRK2) are the major genetic cause of Parkinson's disease (PD), accounting for $\sim 5 \%$ of familial and $0.5-2 \%$ of sporadic cases (Dächsel and Farrer, 2010). Although the strong association between LRRK2 and PD has led to extensive research over the last several years, LRRK2 function in health and disease remains unclear. A recent study by Piccoli et al. (2011) investigated the role of LRRK2 in the control of vesicle dynamics by analyzing mouse primary cortical neuron synapses. The authors showed that silencing LRRK2 in presynaptic neurons altered their ability of to orchestrate excitatory synaptic transmission.

EPSCs evoked in wild-type postsynaptic neurons by depolarization of presynaptic neurons in which LRRK2 was silenced were twice as large as those evoked by depolarization of unsilenced control neurons [Piccoli et al. (2011), their Fig. 2]. This suggested that LRRK2 is involved in the control of vesicle dynamics in presynaptic neurons, which is consistent with data from AlegreAbarrategui et al. (2009), who demonstrated by immunoelectron microscopy that LRRK2 localizes to transmembrane lipid domains, as well as vesicular structures.

Piccoli et al. (2011) used immunostaining against the synaptic vesicle pro-

Received April 19, 2011; revised May 19, 2011; accepted May 23, 2011.

We are grateful for the financial support received from the Michael J. Fox Foundation and the Wellcome Trust/MRC Joint Call in Neurodegeneration award (WT089698).

Correspondence should be addressed to Claudia Manzoni, University College London, Institute of Neurology, Queen Square, WC1N3BG, London, UK.E-mail: c.manzoni@ucl.ac.uk.

DOI:10.1523/JNEUROSCI.1976-11.2011

Copyright $\odot 2011$ the authors $\quad$ 0270-6474/11/319787-02\$15.00/0 tein synaptotagmin to determine that LRRK2-silenced neurons have unusually high numbers of active synapses under resting conditions. Moreover, when cells were depolarized with $\mathrm{KCl}$, which increases vesicle release, no additional increase in synaptotagmin staining was found in these cells [Piccoli et al. (2011), their Fig. 3]. Furthermore, a pulse of hypertonic sucrose, which triggers exocytosis of the entire readily releasable pool of synaptic vesicles (Rosenmund and Stevens, 1996), had no apparent effect in LRRK2-silenced neurons, suggesting that these cells mobilized the maximum number of readily releasable vesicles under resting conditions [Piccoli et al. (2011), their Fig. 8].

Although electrophysiology and immunostaining experiments suggested that more synaptic vesicles were released by LRRK2-silenced neurons, EM analysis showed fewer vesicles in physical contact with the presynaptic membrane [Piccoli et al. (2011), their Fig. 7]. This discrepancy can be explained because the number of docked vesicles does not directly correspond to the readily releasable pool. Additionally this can be justified by looking at the behavior of the wild-type synapse, in which the number of docked vesicles (EM analysis) was higher under basal conditions than after $\mathrm{KCl}$ depolarization, which increased synaptic activity. As the authors suggested, this reduction possibly reflects vesicle depletion through the increase of fusion events upon $\mathrm{KCl}$ depolarization. The same mechanism could then be applied to the LRRK2-silenced synapse: it showed fewer docked vesicles than controls because of an increase in fusion events under resting conditions, thus reconciling EM and electrophysiological/ immunocytochemistry analyses.

In a previous study, Shin et al. (2008) investigated the rate of vesicle endocytosis after vesicle fusion with the synaptic membrane during synaptic activity. Contrary to the results of Piccoli et al. (2011), Shin et al. (2008) found a reduced rate of vesicle recycling upon LRRK2 silencing, suggesting a decrease in synaptic activity. Although the authors of these publications come to different conclusions, we believe the results support the same hypothesis (Fig. 1). Piccoli et al. (2011) described an increase in recycling synapses by examining a moment in time, i.e., by calculating the fraction of all vesicles that were fused with the plasma membrane, as indicated by immunostaining for synaptotagmin without permeabilization. In contrast, Shin et al. (2008) recorded vesicle dynamics by calculating the time needed for fused vesicles to be internalized by using GFP-tagged synaptopHluorin. With this probe, fluorescence is recorded when the GFP tag resides in the extracellular environment but is quenched by the acidic environment within vesicles. The recycling time constants increased when LRRK2 was either silenced or overexpressed. Considering the findings by Shin et al. (2008), we conclude that in LRRK2-silenced neurons, fused vesicles are exposed on the synaptic membrane for longer than they are in control cells. The reduced rate of endocytosis would make vesicle proteins more available to bind an extracellular antibody, as they reside for a longer time on the cell surface. This is in 
perfect accord with the paper by Piccoli et al. (2011), and it is consistent with the decrease in docked synaptic vesicles they detected by EM. Therefore, we believe that the studies by Shin et al. (2008) and Piccoli et al. (2011), through different approaches, point to the same functional conclusion: alteration of LRRK2 levels in the presynaptic terminal impair the normal synaptic activity by reducing endocytosis.

The increased size of EPSCs in postsynaptic neurons after LRRK2 silencing as discussed by Piccoli et al. (2011) could also be explained by a reduced rate of synaptic endocytosis, which might disrupt the clearance of neurotransmitters from the synaptic cleft and thus lead to prolonged excitation of the postsynaptic neuron. Recently, $\alpha$-synuclein has also been associated with neuronal vesicle trafficking (reviewed by Auluck et al., 2010). The fact that two PD-associated genes are linked to vesicle trafficking strongly suggests that such defects contribute to PD pathology. Although it is unclear what causes the age-related pathology and the cell-type-specific degeneration in LRRK2 $\mathrm{PD}$, it is possible that prolonged excitotoxicity renders certain types of neurons more prone to cell death within the aged CNS.

Altered endocytosis could affect other processes in addition to synaptic vesicle dynamics, for example signaling pathways downstream of receptors that require internalization. This is in accordance with data showing that LRRK1, a close family member of LRRK2, is involved in the regulation of epidermal growth factor (EGF) receptor internalization/signaling (Hanafusa et al., 2011), thus influencing downstream cell responses to EGF stimulation.

Although future studies are needed to further clarify the role of LRRK2 in synaptic transmission, the studies by Piccoli et al. (2011) and Shin et al. (2008) suggest the involvement of LRRK2 in regulating membrane dynamics and provide clues about pathological events that might underlie PD.

\section{References}

Alegre-Abarrategui J, Christian H, Lufino MMP, Mutihac R, Venda LL, Ansorge O, WadeMartins R (2009) LRRK2 regulates autophagic activity and localizes to specific membrane microdomains in a novel human genomic reporter cellular model. Hum Mol Genet 18:4022-4034.

Auluck PK, Caraveo G, Lindquist S (2010) $\alpha$-Synuclein: membrane interactions and toxicity in Parkinson's disease. Annu Rev Cell Dev Biol 26:211-233.

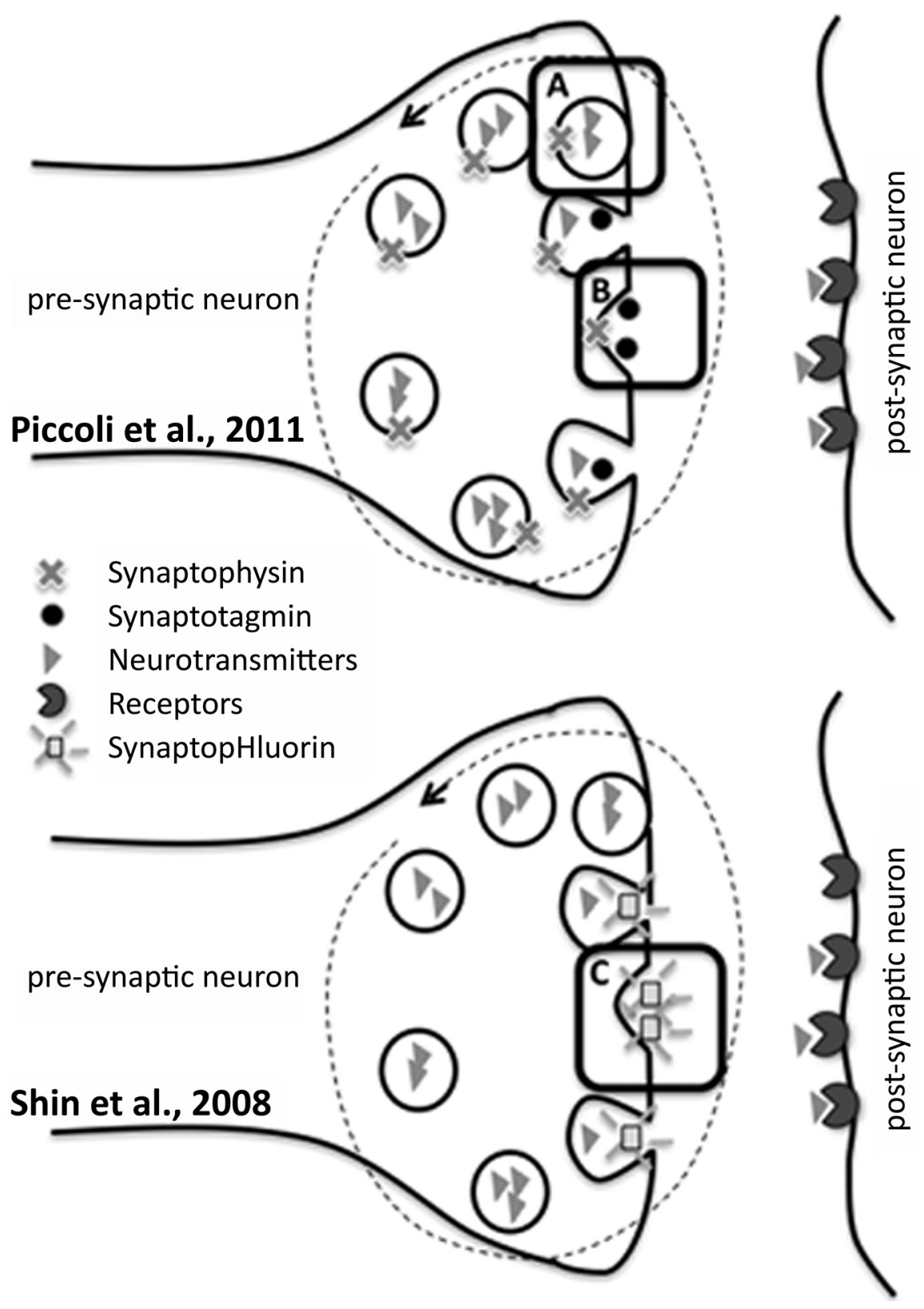

Figure 1. The role of LRRK2 at synapses. A, Piccoli et al. (2011) found that silencing LRRK2 reduced the number of docked vesicles detected by EM compared to wild-type cells. $\boldsymbol{B}$, An increase in the number of recycling synapses was found by immunocytochemistry, suggesting LRRK2 silencing increased synaptic activity. On the other hand, after applying pH-dependent SynaptopHluorin (C), which is detected only when outside acidic vesicles, Shin et al. (2008) found increased fluorescence when LRRK2 levels were altered, suggesting decreased synaptic endocytosis. The reduction of docked vesicles shown in $\boldsymbol{A}$ and the increase in antibody staining as reported in $\boldsymbol{B}$ could both result from the prolonged presence of fused vesicles on the synaptic membrane rather than an increase in the total number of fusion events, thus reconciling the results of Piccoli et al. (2011) and Shin et al. (2008).

Dächsel JC, Farrer MJ (2010) LRRK2 and Parkinson disease. Arch Neurol 67:542-547.

Hanafusa H, Ishikawa K, Kedashiro S, Saigo T, Iemura S, Natsume T, Komada M, Shibuya H, Nara A, Matsumoto K (2011) Leucine-rich repeat kinase LRRK1 regulates endosomal trafficking of the EGF receptor. Nat Commun 2:158.

Piccoli G, Condliffe SB, Bauer M, Giesert F, Boldt K, De Astis S, Meixner A, Sarioglu H, VogtWeisenhorn DM, Wurst W, Gloeckner CJ, Matteoli M, Sala C, Ueffing M (2011) LRRK2 controls synaptic vesicle storage and mobilization within the recycling pool. J Neurosci 31:2225-2237.

Rosenmund C, Stevens CF (1996) Definition of the readily releasable pool of vesicles at hippocampal synapses. Neuron 16:1197-1207.

Shin N, Jeong H, Kwon J, Heo HY, Kwon JJ, Yun HJ, Kim CH, Han BS, Tong Y, Shen J, Hatano T, Hattori N, Kim KS, Chang S, Seol W (2008) LRRK2 regulates synaptic vesicle endocytosis. Exp Cell Res 314:2055-2065. 